\title{
DESAFÍOS DE LA TERAPIA OCUPACIONAL EN LA INCLUSIÓN SOCIAL DE JÓVENES INFRACTORES DE LEY, EN MEDIOS DE RÉGIMEN CERRADOS DE INTERNACIÓN EN CHILE.
}

\author{
CHALLENGES OF OCCUPATIONAL THERAPY IN THE SOCIAL \\ INCLUSION OF YOUNG OFFENDERS IN CLOSED CENTERS IN CHILE.
}

\author{
Irene Muñoz E. ${ }^{1}$ Rodrigo Goycolea $M .^{2}$
}

\section{RESUMEN}

La presente investigación de carácter cualitativo y exploratorio, tuvo por objetivo desarrollar un análisis de las acciones que un grupo de terapeutas ocupacionales realizan en los programas de reinserción social de jóvenes infractores de ley, en centros de régimen cerrado y de internación. Para esto, se contactó a un grupo de 29 profesionales que contestaron un cuestionario semiestructurado. El análisis de los hallazgos se realizó empleando la técnica del análisis de contenido. Dentro de los resultados se identificó que las acciones profesionales se orientan a las necesidades ocupacionales de los adolescentes, enfocadas hacia la participación social e inclusión laboral. Las intervenciones se concentran en los procesos motivacionales y de responsabilización, con eje en el vínculo terapéutico, la entrevista motivacional y la consideración de los intereses de los adolescentes. Se estima que las rutinas al interior del centro y el área educacional no son abordadas, en su mayoría, por los terapeutas ocupacionales. Los equipos interdisciplinarios de los centros han incorporado el rol de Terapia Ocupacional con dificultades y desconocimiento, atribuyéndoles cierta experticia solamente en las áreas de capacitación y en el ámbito laboral.

\footnotetext{
${ }^{1}$ Magíster en Educación en Ciencias de la Salud. Licenciada en Ciencias de la Ocupación Humana. Terapeuta Ocupacional. Universidad de Chile. Orientadora en Relaciones Humanas y Familia Instituto Profesional Carlos Casanueva. Profesor Asociado Facultad de Ciencias de la Salud. Escuela de Terapia Ocupacional. Universidad Central de Chile. Lord Cochrane 417. Torre A. Piso 5. Teléfono (56 2) 25826388 email: imunozespinosa@gmail.com irenemunoze@ucentral.cl

${ }^{2}$ Magister en Drogodependencias, Universidad de Barcelona, España. Licenciado en Ciencias de la Ocupación Humana. Terapeuta Ocupacional Universidad de Playa Ancha. Valparaíso. Académico Facultad de Ciencias de la Salud. Escuela de Terapia Ocupacional. Universidad Central de Chile. Lord Cochrane 417. Torre A. Piso 5. Teléfono (56 2) 25826388 email: rodrigo.goycolea@gmail.com rodrigo.goycolea@ucentral.cl
} 
Concluimos que existen distintos niveles de desarrollo profesional en el área, ya que no todos los profesionales presentan experiencia de trabajo con población adolescente privada de libertad, los cuales presentan altos niveles de complejidad psicosocial. Existen avances en propuestas de diagnóstico ocupacional del adolescente infractor, sin embargo se requiere generar evidencia en lo que respecta a intervenciones específicas que fortalezcan acciones que mejoren el desempeño ocupacional de los adolescentes para lograr procesos de reinserción efectivos.

Palabras claves: Adolescentes, Infractores de Ley, Terapia Ocupacional, Inclusión Social

\begin{abstract}
The research study has a qualitative and exploratory approach and aimed to develop an analysis of the actions made by a group of occupational therapists within social reintegration programs for young offenders in closed centers. For this, we contacted a group of 29 professionals who answered a semi-structured questionnaire. The analysis of the findings was performed using the content analysis method. The results identified that professional actions are oriented towards young' occupational needs, focused towards social participation and work inclusion. The interventions focus on motivational processes and accountability, with emphasis in the therapeutic relationship, motivational interviewing and consideration of the interests of teenagers. It is estimated that the teenagers' routines in the center and the area of education are mostly not addressed by occupational therapists. Centers' Interdisciplinary teams have incorporated the role of Occupational Therapy with difficulties and lack of knowledge, attributing them only certain expertise in the areas of workplace training.

We conclude that there are different levels of professional development in the area, since not all professionals have experience working with teenagers deprived of freedom, which challenges with high levels of psychosocial complexity. There are advances in diagnosis of occupational proposed juvenile offenders, but is necessary to generate evidence regarding specific interventions to strengthen actions to improve teenagers' occupational performance to achieve effective reintegration processes.
\end{abstract}

Keywords: Young Offenders, Occupational Therapy, Social Inclusion 


\section{INTRODUCCION}

Desde la entrada en vigencia de la Ley N 20.084 de Responsabilidad Penal Adolescente en Chile en el año 2007, cuya normativa estableció un sistema especial de justicia penal para los jóvenes mayores de 14 años y menores de 18 años se generó el propósito reformar radicalmente la respuesta del Estado ante los actos que revisten carácter de crimen o simple delito cuando ellos son cometidos por personas menores de 18 años de edad. En este contexto se asumen los mandatos de la Convención Internacional sobre Derechos del Niño y del adolescente y se inicia la convicción de que los/las adolescentes infractores de ley son sujetos de derechos, por lo tanto requieren de protección integral y acceso a procesos de reinserción que tiendan no sólo a que el/ la adolescente asuma la responsabilidad por sus actos, sino que, al mismo tiempo, reciba una intervención susceptible de educarlo respecto de la responsabilidad y orientada al logro de su integración social (SENAME, 2011). A su vez, la implementación de la intervención tendrá una mirada complementaria desde la perspectiva ecosistémica, con el fin de abordar de manera integral todos los ámbitos de intervención, visualizando al individuo, la familia y la comunidad como un todo relacionado, organizando a las diferentes instituciones y profesionales para efectuar una intervención coherente y coordinada en torno a un objetivo común (Barudy, 2001).

En el proceso socioeducativo necesario de implementar con los/las adolescentes se destacan cuatro componentes: Responsabilización, Reparación, Habilitación y Reinserción social, los cuales constituyen un todo sistémico que implica el consolidar modelos de intervención cada vez más integrados, multidisciplinarios e intersectoriales. Es en este desafío, que se requiere equipos constituidos por profesionales competentes, con claridad en la definición de sus roles y competencias al servicio del proceso de inclusión social.

En este marco, Terapia Ocupacional posee una escasa evidencia de las acciones realizadas en personas privadas de libertad; y aún más, en la población infantoadolescente de medios con régimen cerrado e internación pertenecientes al Servicio Nacional de Menores en Chile, en adelante SENAME. Es por esto, que esta investigación tiene como propósito reconocer el rol que la Terapia Ocupacional desempeña en los actuales programas de rehabilitación, reinserción e inclusión de social de los adolescentes infractores de ley.

Terapia Ocupacional es una profesión relativamente joven, y hasta la fecha, su participación en el sistema de justicia es limitada no sólo a nivel nacional, sino que también a nivel internacional. En publicaciones de Few, J., \& Charles, K. (2001) se evidencia la importancia de que la profesión se incorpore en los procesos de rehabilitación de adolescentes con problemas con la justicia, mencionándose algunas orientaciones con respecto a la realización de programas con metas, estrategias y métodos medibles de evaluación. Se destaca como acción el desarrollo de programas de habilidades para la vida, los cuales favorecen la adquisición de habilidades que les permiten a los/ las adolescentes cumplir con las ocupaciones más significativas para su ciclo vital (educación - trabajo, ocio, juego, participación social autocuidado y descanso) de una manera socialmente aceptable y auto-gratificante). 
En un estudio realizado por Farnworth (2000), se sugirió que los jóvenes infractores de ley participan en una alta proporción de ocupaciones no estructuradas, y que este factor podría afectar a su salud y bienestar, impactando negativamente en su futuro, específicamente en el desarrollo de habilidades para la vida independiente. Se concluye que muchos jóvenes se beneficiarían de rutinas estructuradas con respecto a las actividades que desean y les sean significativas, por lo que el favorecer un ambiente estructurado es importante para un desempeño ocupacional satisfactorio a futuro.

En Chile hay muy pocos reportes acerca de experiencias en el ámbito de la rehabilitación con jóvenes infractores de ley, existiendo escasa evidencia de evaluaciones sistemáticas a través del tiempo, siendo más limitada la información relacionada con la intervención en un régimen cerrado (Poblete, 2014).

El SENAME incluye en sus perfiles de cargos el que compete al Terapeuta Ocupacional, en el cual se describe que el cargo tiene como propósito principal: "Aplicar correctamente técnicas, métodos y actuaciones que favorecen respecto de los/as jóvenes privados de libertad, la restauración de funciones, la superación de déficit, la evaluación y significación de supuestos comportamentales, mediante el uso terapéutico de actividades de diversa índole, para el logro de mayores niveles de autonomía e independencia, conducentes a una exitosa reinserción en todos los ámbitos de la vida, físico, mental, laboral y social, focalizándose este cometido en la formación laboral, empleabilidad y emprendimiento". ( $\mathrm{p} 27)$

Este perfil de cargo incluye una serie de funciones claves tendientes a:

- Evaluación y valoración de las capacidades y habilidades funcionales que presentan los adolescentes privados de libertad, proyectando potencialidades y objetivos de trabajo consecuentes.

- Evaluación de las limitaciones de cada adolescente y formulación de un plan de compensación.

- Elaboración y evaluación de programas de tratamiento de rehabilitación con sus correspondientes objetivos individualizados y diferenciados.

- Entrenamiento y reeducación en el uso de habilidades básicas e instrumentales para la vida diaria.

- Confección, prescripción y operacionalización de apoyos técnicos, entrenamiento del/la adolescente y asesoría a los equipos de intervención directa.

- Formulación de indicaciones para otros programas que conformen la oferta socioeducativa del/la adolescente, estableciendo indicadores de riesgo, avance y logro.

- Formulación de propuestas para la superación de obstaculizadores en el desarrollo de la oferta regular. 
- Asesoramiento a familias, equipo de intervención directa, profesionales de otras especialidades.

- Capacitación a los /las adolescentes en temáticas específicas para lograr un mayor grado de reinserción socio-laboral posible (habilidades blandas por ejemplo).

- Diagnosticar individualizada y diferenciadamente los puestos de trabajo y entorno.

- Planificar y/u operacionalizar el proceso de orientación, capacitación y/o ubicación laboral de los/las jóvenes.

- Formulación y/o gestión de propuestas de colaboración con instituciones públicas y/o privadas para la promoción y desarrollo de programas de reinserción socioeducativa $\mathrm{y} / \mathrm{o}$ sociolaboral para los jóvenes (programas de acompañamiento educativo y/o laboral).

De acuerdo lo descrito, creemos que la labor de los/las terapeutas ocupacionales que se desempeñan en los Centros de Internación Provisoria (CIP) y en los Centros de Régimen Cerrado (CRC) del país, debería estar orientada además a la evaluación y estructuración de las rutinas de los adolescentes infractores de ley, considerando las actividades de la vida diaria básicas, instrumentales y avanzadas, trabajo, educación, juego, tiempo libre, ocio y participación social. Lo anterior, se fundamenta en que Terapia Ocupacional posee las herramientas necesarias para hacer de las rutinas actividades organizadas y relevantes, dentro del contexto institucional, de acuerdo a las motivaciones y necesidades de los/ las adolescentes, considerando diferenciaciones y especificidades de cada uno, donde el análisis de las actividades y del ambiente influye en el desempeño de ellas. Otro aspecto importante, sería el favorecer el desempeño ocupacional del/la adolescente, la restauración de habilidades y patrones de desempeño ocupacional, específicamente de sus roles y la re-significación de supuestos comportamentales, mediante la apropiación de rutinas significativas y el uso terapéutico de actividades de diversa índole para el logro del equilibrio ocupacional, incorporándose mayores niveles de autonomía que favorezcan una proyección ocupacional pro-social, en coherencia con el proyecto de vida elaborado con el/la adolescente.

En este contexto de intervención profesional cobra relevancia el concepto de Justicia Ocupacional, que se entiende como "la promoción de un cambio social y económico para incrementar la conciencia individual, comunitaria y política, los recursos y la igualdad de oportunidades para el desarrollo de ocupaciones que permitan a las personas alcanzar su potencial y experimentar bienestar" (Wilcock and Townsend, 2004 p. 80). Estas autoras señalan que existen tres tipos de injusticia ocupacional:

1.- Privación Ocupacional: Sucede cuando existe una privación tanto de recursos como de oportunidades para realizar una ocupación. La injusticia crece cuando las sociedades toleran este tipo de privación, mientras otras personas cuentan con los recursos para realizar estas ocupaciones y las desempeñan. Podríamos reflejar este concepto en la historia de los/las adolescentes antes del ingreso a un CIP-CRC y luego cuando ingresan este principio se encuentra en tensión ente la privación de las oportunidades dentro de un sistema privativo de libertad.

2.- Marginación Ocupacional: Es el resultado de experiencias de vida sin sentido ni propósito. La carencia de vivencias positivas en una ocupación puede distorsionar la formación de la identidad. 
Esto corresponde principalmente a ocupaciones muy estandarizadas, repetitivas, sin oportunidad para poder elegir dentro de ésta. El hecho de que algunas personas sean privilegiadas mientras otras son enajenadas, regimentadas, limitadas y posiblemente explotadas en actividades diarias de trabajo hace que esto se convierta en una materia de justicia. Ejemplo de esto, es lo referido al ámbito laboral en la cual las características de ciertos empleos, más allá de su valor económico, pueden enriquecer a las personas mental y espiritualmente, mientras que otras ocupaciones laborales son experimentadas por algunas o todas las personas como aburridas o faltas de sentido. Esto si lo aplicamos a la fuerza laboral de los/las adolescentes que con sus antecedentes educacionales, laborales más sus antecedentes delictuales se encuentran en una situación de marginalidad que es todo un desafío para los profesionales que se desempeñan con esta población, disminuir estos niveles de marginalidad y favorecer oportunidades.

3.- Desequilibrio Ocupacional: Corresponde a una categoría temporal, basándose en el razonamiento que relaciona la salud humana y el bienestar con variaciones productivas y tiempo de ocio. La clasificación jerárquica de las ocupaciones conduce a un mercado laboral en el cual aquellos con habilidades y conocimientos particulares son remunerados y tienen un acceso a trabajo, mientras que otros no son capaces de encontrar uno. El resultado es un crecimiento ocupacional desequilibrado en el cual algunas personas están sobrepasadas o sobrecargadas ocupacionalmente, y otras están desocupadas o carentes de ocupación. Largas jornadas de trabajo, durante muchos años de la vida, en desmedro de otras áreas de desempeño, tales como tiempo libre, es uno de los ejemplos de desequilibrio ocupacional que viven muchas personas a diario, o en estos casos un exceso de tiempo libre frente a actividades educacionales o laborales.

Si relacionamos los conceptos anteriores con el comportamiento delictual y específicamente con la Inclusión Social como objetivo, podemos identificarlos como factores críticos que inciden en la participación en ocupaciones por parte de los/las adolescentes. Las oportunidades que eventualmente podría presentar el ambiente, no están dadas de manera igualitaria para todos los individuos de esta sociedad, incluso entre los que están "excluidos" (Paulson, 1980). Para ejemplificar esta situación basta pensar en el abordaje que se realiza con la población de adolescentes sin ningún otro condicionante, frente al adolescente infractor de ley, ambos deberían estar insertos en el sistema educacional formal, si bien cada uno debe enfrentar obviamente sus propias particularidades, la carga social y moral negativa del segundo individuo es mayor, debido a la relación que se hace con la delincuencia y por lo tanto, el esfuerzo de integración sociolaboral, desde este escenario, tiene mayores niveles de complejidad. Con el ejemplo anterior se muestra el hecho de cómo la sociedad establece y deposita distintos estigmas en distintos tipos de personas consideradas "excluidas" y asimismo, las oportunidades para enfrentar esta situación de "exclusión" están limitadas según las condiciones y trayectorias de vida (experiencias) que ellos presentan (Kruse, 2011). 
Si los Terapeutas Ocupacionales no cuestionamos con firmeza y construimos evidencia para el funcionamiento de la "sociedad" y asumimos una visión funcionalista de ésta, corremos el riesgo de ser meros profesionales que repetimos lo que se hace, que incluso guían su práctica, probablemente de manera inconsciente, a perpetuar y reafirmar el modelo social "exclusivo y privilegiado" de algunos, en donde la mayoría de nosotros, por distintas razones, no cabe. Con lo anterior, se destaca que la práctica y rol del Terapeuta Ocupacional con los adolescentes infractores de ley, al momento de hablar de Inclusión Social, debe enfocarse no sólo a la intervención con la persona, sino también sobre la sociedad, intencionando que ésta permita y promueva la participación social, política y económica de nuestros usuarios, que puedan experimentar un rol laboral y educacional activo a través del ejercicio de sus derechos y obligaciones y en consecuencia, tener la alternativa y posibilidad de conseguir, mantener y progresar en un empleo.

Frente a la poca evidencia del rol que compete a la Terapia Ocupacional en esta área, y en relación a lo que se espera por parte del SENAME de ser un profesional que aporte a los programas de reinserción socioeducativa y/o sociolaboral de los adolescentes infractores de ley, es que se justifica el desarrollo de esta investigación, orientada a desarrollar un análisis crítico de las acciones de Terapia Ocupacional que se están realizando actualmente en los programas de reinserción social de adolescentes infractores de ley en los regímenes cerrados y de internación provisoría a lo largo del país.

\section{METODOLOGÍA}

Esta investigación se llevó a cabo bajo un enfoque de tipo cualitativo y exploratorio ya que se desarrolló un proceso inductivo de exploración y descripción que permitió interpretar algunos hallazgos que facilitaron el desarrollo de nuevas hipótesis de investigación. El objetivo fue abordar las acciones de terapia ocupacional en el ámbito de la intervención de adolescentes infractores de ley, tema poco estudiado del cual no se registran evidencias, con el fin de avanzar en la práctica de la disciplina (Hernández, 2007).

La recolección de los datos consistió en obtener las perspectivas y puntos de vista de los y las terapeutas ocupacionales que se encontraban trabajando en los CIP-CRC del SENAME a través de un muestreo por conveniencia, en el cual se contactó a un grupo de terapeutas ocupacionales voluntarios que se encontraban desempeñándose en los centros de internación provisoria y centros de régimen cerrado de adolescentes infractores de ley que quisieran aportar al estudio. También se complementó con un muestreo en bola de nieve, proceso en el cual se les solicitó a los informantes que recomendaran a otros posibles participantes en el estudio. Lo anterior se justificó debido al desconocimiento del número total de terapeutas ocupacionales que se encuentran trabajando en el área.

La información se obtuvo a través de un cuestionario semi-estructurado autoadministrado de manera electrónica a 29 terapeutas ocupacionales vinculados a los centros nombrados anteriormente 
donde se encuentran los/las adolescentes infractores de ley adscritos a SENAME, quienes mediante un consentimiento informado accedieron a participar del estudio.

Para esta investigación se planteó como objetivo general el desarrollar un análisis de las acciones de Terapia Ocupacional en los programas de reinserción social de jóvenes infractores de ley.

Objetivos específicos:

1. Describir las acciones que desempeñan los terapeutas ocupacionales que forman parte de los equipos de reinserción de adolescentes en los centros de régimen cerrados adscritos a SENAME

2. Conocer las percepciones de los/las terapeutas ocupacionales en cuanto a sus competencias profesionales que les permiten desempeñarse en forma eficiente en los programas de reinserción social de adolescentes infractores de ley.

3. Conocer los desafíos de la profesión en el contexto de los procesos de inclusión social de adolescentes infractores de ley.

El proceso de producción de información se realizó a través de la técnica de análisis de contenido. La técnica utilizada fue el desarrollo de categorías deductivas, las que se formularon a partir de la teoría, después se construyeron paso a paso los códigos los que se aplicaron a los textos. La lectura se realizó siguiendo el método científico y combinó intrínsecamente, la observación y producción de los datos, y la interpretación de los mismos. (Abela, 2002). Se establecieron categorías temáticas para el análisis de las respuestas, siendo estas significativas y excluyentes para el objetivo del estudio: Acciones que desempeñan los terapeutas ocupacionales en los CRC; Competencias profesionales que les permiten desempeñarse en los CRC; Desafíos de la profesión en el contexto de los procesos de inclusión social de adolescentes infractores de ley

\section{RESULTADOS:}

El análisis cualitativo de los cuestionarios contestados por los/las terapeutas ocupacionales participantes fue llevado a cabo a través de la codificación, es decir, a partir del establecimiento de categorías que emergieron desde la teoría para la identificación de unidades de análisis en el texto. Los resultados serán expuestos en siete apartados:

- Modelos o enfoques profesionales

- Acciones de Terapia Ocupacional

- Percepción acerca de la efectividad de los procesos de reinserción. 
- Percepción acerca de las ocupaciones que se requieren fortalecer.

- Herramientas profesionales que permiten favorecer los procesos motivacionales.

- Percepciones en relación al rol de Terapia Ocupacional en los equipos.

- Aportes de la profesión a los actuales programas de reinserción social.

Asimismo, por cada categoría se expondrá el proceso interpretativo desarrollado por el equipo investigador.

Del total de terapeutas ocupacionales que participaron del estudio un $74 \%$ fueron mujeres y un $26 \%$ hombres, siendo el promedio del grupo participante 29 años de edad. Todos con título profesional universitario, con un promedio de 5 años de egreso, siendo la con mayor años de ejercicio profesional una terapeuta ocupacional egresada el año 2001 y la con menor experiencia una egresada el año 2013. El 100\% de ellos trabaja o había trabajado hasta hace un año atrás en alguno de los CIP CRC de SENAME, siendo la antigüedad promedio en el cargo 13,5 meses, los que varían entre 36 meses el más antiguo y un mes la más nueva en el cargo.

\section{Modelos o enfoques profesionales utilizados en los procesos de intervención con adolescentes infractores de ley.}

Los/las terapeutas ocupacionales refieren trabajar explícitamente con el Modelo de Ocupación Humana el cual se repite en la mayoría de los participantes, lo anterior reflejaría que lo consideran un modelo factible de aplicar a la realidad contextual de los centros de internación de régimen cerrado. Algunos, en menor grado mencionan complementar las intervenciones con el Marco de Trabajo de la Asociación Americana de Terapeutas Ocupacionales, el Modelo cognitivo conductual, el Modelo ecológico y sistémico, el Modelo de redes, el Enfoque de Educación Popular, el Modelo Transteórico del cambio y el Modelo Canadiense del Desempeño Ocupacional. Llamando la atención que sólo un profesional considera en su intervención el enfoque de derechos humanos, que es la guía que debieran tener las intervenciones de acuerdo a las orientaciones técnicas del SENAME.

\section{Acciones de Terapia Ocupacional que aportan a las necesidades ocupacionales de los adolescentes infractores de ley en los CIP-CRC.}

Se describen que las acciones desarrolladas desde la profesión, aportan a las necesidades ocupacionales que presentan los adolescentes, desde la justificación que Terapia Ocupacional es una disciplina holística, que considera no sólo las necesidades si no también las fortalezas, habilidades y competencias de los adolescentes, como base del proceso de intervención. A la vez, se considera que la ocupación es parte vital de la salud para el funcionamiento autónomo y satisfactorio de cualquier ser humano; sea cual sea su etapa o ciclo vital. Sin embargo, muchas veces se reconoce que el sistema impide avanzar o intervenir en las diferentes áreas de desempeño del joven, considerando el conjunto de sus intereses y motivaciones. 
Se destaca que se pretende favorecer los niveles de participación ocupacional de los jóvenes al interior de los centros, de acuerdo a las expectativas que tienen los adolescentes de ellos mismos. Consecuentemente, se evalúan las dificultades específicas en el desempeño del/la adolescente que pueden ser trabajadas en vista de esas expectativas y como fin último de favorecer el bienestar y calidad de vida de cada uno de ellos. Una de las mayores dificultades que se observa en los/las adolescentes, es la alteración de sus componentes volitivos, lo cual se traduce en un desequilibrio ocupacional importante. De modo que favorecer el auto-concepto y la auto-eficacia percibida, le permitirían retomar ocupaciones acordes a sus objetivos e intereses, principalmente del tipo prosociales.

Se insiste en que el/la terapeuta ocupacional puede desarrollar acciones específicas para fortalecer el desempeño ocupacional de los adolescentes y no necesariamente las acciones se deben centrar netamente en el aspecto de capacitación y/o habilitación laboral, como en muchos servicios es identificada su acción profesional.

\section{Percepción acerca de la efectividad de los procesos de reinserción de adolescentes infractores de ley.}

Los/las terapeutas ocupacionales refieren que los lineamientos técnicos están enfocados solamente hacia al plano laboral y no consideran las necesidades ocupacionales reales acordes al ciclo vital del adolescente. Se reconoce que en la actualidad desde Terapia Ocupacional, se está implementando un proceso diagnóstico donde se están ejecutando acciones de intervención y de mejora constantes, que permitan retroalimentar las orientaciones técnicas. Se menciona que existen muchas variables a considerar, como coordinaciones y estrategias entre las medidas implementadas y los programas establecidos los que se centran más bien aspectos teóricos, sin plasmarse en la factibilidad práctica de acuerdo a los distintos contextos de los centros.

Se destaca que la complejidad de este grupo, en cuanto a las trayectorias de vulneración de derechos y secuencias de delitos, sobrepasa a las competencias de los programas y a los objetivos por los cuales fueron creados, respecto a su capacidad para abordarlos, el tiempo necesario para hacerlo y la capacidad de una red socio sanitaria integrada para apoyarlos. A la vez los resultados de la intervención, muchas veces, no pueden ser cuantificables, por el nivel de complejidad del problema. A la etapa de vida de los adolescentes, se le suma el contexto socio-cultural en el cual se han desarrollado, el cual ha fomentado un esquema valórico antisocial lo que es difícil de resignificar. Por lo tanto, no siempre los resultados positivos pueden ser juzgados por la cantidad de los adolescentes que se reinserta socio-familiar, educativa y laboralmente en el corto plazo, sino que se logra, por el cambio en su estilo de pensamiento con respecto a si mismo y a los que les rodean, es decir, en aspectos valóricos, morales y éticos. 
También se menciona que la efectividad, es algo cuestionable, cuando la base es una solución punitiva más que de reinserción, la efectividad de la reinserción siempre va a estar ligada, con la experiencia social, de acuerdo a los informantes; por lo que todo sistema que prive al joven de un ambiente social, como lo son los sistemas cerrados, va a lograr un menor impacto de las alternativas de reinserción en el joven y su esperado proceso de inclusión social.

\section{Percepción acerca de las ocupaciones que se requieren fortalecer en los adolescentes infractores de ley.}

Se menciona que las ocupaciones relacionadas con la participación social, el ocio y el tiempo libre, las actividades instrumentales de la vida diaria y el trabajo, se requieren fortalecer. También es necesario considerar el retomar el rol de estudiante, el cual muchas veces ha sido abandonado por no ser significativo para el/la adolescente ni para su familia, en este contexto se hace necesario más que incluirse a ámbitos escolares tradicionales, los cuales no están preparados para recibir a estos jóvenes, se requieren mayores instancias de capacitación en oficios con certificación, sin la obligatoriedad de cursar educación formal

A la vez el fortalecimiento de patrones de desempeño como hábitos y rutinas estructuradas, junto al ejercicio de roles que potencien una identidad prosocial, son elementos centrales a abordar en los procesos de intervención. Clave es el desarrollo de las habilidades de interacción social, por sobre todo habilidades blandas tales como el respeto, la empatía, la asertividad, entre otras, que con su adquisición permitirían avanzar hacia objetivos superiores.

\section{Herramientas profesionales que permiten favorecer los procesos motivacionales y la responsabilización del adolescente infractor de ley.}

Los/las terapeutas ocupacionales reconocen en su mayoría la entrevista motivacional como eje de la intervención, la identificación y validación de los intereses propios del joven que sean el motor de un nuevo proyecto de vida hacia el medio libre. Las habilidades terapéuticas que más se ponen en juego durante estos procesos son la asertividad, la escucha activa, el enfoque centrado en los recursos del joven, la tolerancia, la empatía, conjugadas muchas veces con episodios críticos en que se requieren estrategias de contención y de manejo de crisis.

El análisis de actividad es clave para determinar las exigencias de las actividades que se le proponen a los jóvenes, de modo de no causar frustraciones innecesarias, lo que interfiere en los procesos motivacionales.

En cuanto a la responsabilización, es el vínculo terapéutico el que permite establecer diálogos reflexivos en forma individual y en dinámicas grupales en los cuales se problematizan las distintas situaciones, en las cuales los/las adolescentes analicen y empaticen con la vulneración de derechos de terceros. 


\section{Percepciones en relación al rol de Terapia Ocupacional en los equipos.}

Se menciona que la incorporación del terapeuta ocupacional ha sido con cierta resistencia por parte de los profesionales y técnicos, debido a que se asume que la profesión sólo aborda el plano laboral, esto porque los lineamientos establecidos en las orientaciones técnicas de SENAME así lo establecen. Se reconoce el rol dentro del proceso de apoyo, monitoreo y colocación laboral de los jóvenes, mediante un acompañamiento permanente a los adolescentes antes, durante y hasta finalizar el plan de intervención o la condena privativa de libertad. Se considera como relevante, la participación activa de los/las terapeutas ocupacionales en la creación de bases técnicas para licitación de capacitaciones laborales para los/las adolescentes, trabajando en equipo con el personal administrativo de los centros.

Ha habido avances debido a que antes se asociaba al rol de "tallerista" o "monitor de manualidades". El desafío es socializar la importancia que la acción profesional se orienta al desarrollo de todas las ocupaciones del ser humano y puede aportar en distintas etapas del proceso de reinserción del/la adolescente.

\section{Aportes de la profesión a los actuales programas de reinserción e inclusión de social de los adolescentes infractores de ley.}

Los aportes se centran en incorporar la visión de la ocupación como fuente de sentido, significado y propósito en todo ser humano, permitiéndole al joven incorporar la ocupación como un medio terapéutico o como un fin en si mismo y por supuesto que el joven incorpore un proyecto de vida que sea adaptativo y reconocido socialmente.

Un aporte característico es el hecho de ser profesionales con competencias para evaluar y desarrollar las habilidades de desempeño ocupacional en forma integral, que son las unidades básicas, junto a los factores de las persona, para que éstas puedan llegar a desempeñar ocupaciones significativas. Lo anterior, entrega una mirada complementaria que enriquece los procesos diagnósticos de otras profesiones. En base a esto, otorgamos múltiples recursos en cuanto a visibilizar oportunidades de cambio y habilidades de los/las adolescentes que pueden ser potenciadas para el alcance de los objetivos propuestos, a través de metodologías de trabajo adecuadas a la población adolescente. 
Otro aporte es la capacidad de realizar análisis del desempeño ocupacional, en conjunto con el análisis y los contextos de desempeño que influyen en los comportamientos ocupacionales que tienen y requieren modificar los jóvenes infractores.

\section{DISCUSIÓN.}

Se ha detectado que existen distintos niveles de desarrollo profesional en el área, ya que no todos los profesionales presentan experiencia de trabajo con población adolescente, privada de libertad con altos niveles de complejidad. Existen avances en propuestas de diagnóstico e intervención ocupacional del/la adolescente infractor, sin embargo llama la atención que los terapeutas ocupacionales no mencionan el desarrollo y aplicación de la herramienta de Evaluación Diferencial Individual, instrumento creado por la dirección nacional del SENAME y que es una indicación que todo terapeuta ocupacional debe aplicar sin embargo los participantes que contestaron el cuestionario, no la señalan como una de sus funciones. Este instrumento considera en uno de sus apartados el área laboral que es la sección donde interviene el terapeuta ocupacional.

Sin duda, una de las funciones más relevantes es el favorecer la estructuración de la rutina del adolescente en los centros, acción que no es realizada por la totalidad de los participantes del estudio, siendo un desafío a que se incorpore como eje central del proceso de intervención. Son los/las terapeutas ocupacionales los más idóneos en evaluar la rutina para cada adolescente y del centro con la finalidad de considerar los intereses de los mismos, en conjunto con la oferta programática y los recursos materiales y humanos, necesarios para que sean satisfactorias, es tan relevante el tema de la rutina que debe ser aprobado por la Seremi de Justicia de cada región considerando la oferta programática.

En la actualidad los terapeutas ocupacionales se encuentran en etapa de exploración respecto a los distintos instrumentos de evaluación y diferentes intervenciones, ya sea por los distintos contextos, cantidad de adolescentes privados de libertad, si son adolescentes condenados o imputados, hombres o mujeres, mayores o menores de edad, etc.

Los/as terapeutas ocupacionales reconocen que sus evaluaciones e intervenciones deben ser consideradas en una mirada amplia no solo considerarlos en el ámbito laboral, sino en todas las ocupaciones descritas para el ciclo vital adolescente.

En cuanto a poder sensibilizar con respecto al rol profesional, se requiere un protagonismo activo de los/las terapeutas ocupacionales en las reuniones de equipo con los otros profesionales, tales como psicólogos, trabajadores sociales, profesores y técnicos.

Sin duda, los/ las terapeutas ocupacionales que fueron parte de este estudio han realizado un importante camino de desarrollo profesional, involucrándose en un área de intervención que no tiene evidencia que sustente su actuar, sin embargo han logrado progresivamente ir mostrando el 
importante trabajo desarrollado, muchas veces a pulso y con lineamientos que no necesariamente comprenden nuestro complejo actual profesional.

El desafío es generar evidencia en lo que respecta a evaluaciones e intervenciones específicas que fortalezcan acciones que mejoren el desempeño ocupacional de los adolescentes para lograr procesos de reinserción efectivos y que impliquen un trabajo integral junto al resto de los profesionales que aportan en desde sus disciplinas a este complejo proceso social. 


\section{Referencias Bibliográficas:}

Abela, J. A. (2002). Las técnicas de análisis de contenido: Una revisión actualizada. $<$ http://public.centrodeestudiosandaluces.es/pdfs/S200103.pdf

Barudy, J. (2001). El tratamiento de familias en donde se producen abusos y malos tratos infantiles. Jornadas de trabajo sobre el tratamiento familiar en situaciones de malos tratos y abuso en la infancia, Mallorca.

Farnworth, L. (2000). Time use and Leisure Occupations of Young Offenders. The American Journal of Occupational Therapy, volumen 54, (N³), p 315-p325.

Few, J., \& Charles, K. (2001). What can occupational therapy offer the youth justice service? A review of a pilot placement of occupational therapy students at the youth justice service (townsville/Thuringia). Australian Institute of Criminology. Volume 1, ( $\left.{ }^{\circ} 1\right), \mathrm{p} 1-\mathrm{p} 8$.

Hernández Sampieri, Roberto. (2007) Metodología de la Investigación $5^{\circ}$ Ed. México, Editorial: McGraw-Hill

Kruse, J. (2011). Occupational engagement may be effective in reducing recidivism among juvenile offenders. Emergin Practice, CATs, $p-7$.

Ley 20.084 (2005) www.bcn.cl/leyes/pdf/actualizado/244803.pdf

Paulson, C. (1980). Juvenile Delinquency and Occupational Choice. The Occupational Theraphy Behavior in Pediatrics. volumen 34 ( $\mathrm{N}^{\circ}$ 9), p 564-p571.

Poblete, M. 2014. Identificación delictual juvenil: una propuesta de intervención ocupacional. Revista Chilena de Terapia Ocupacional. Vol. 14. N¹ Pág. 45-52. 
Servicio Nacional de Menores. (2011) Guía de evaluación diferenciada individual, para los centros cerrados de administración directa. Departamento de Justicia Juvenil. Santiago, Chile.

Servicio Nacional de Menores. (2011) Guía para el manejo de caso en centros de cumplimiento de condena en régimen cerrado con programa de reinserción social, documento de trabajo. Departamento de Justicia Juvenil. Santiago, Chile.

Servicio Nacional de Menores. Departamento de justicia juvenil. (2011) Orientaciones técnicas para la intervención Centros de cumplimiento de condena Régimen cerrado con programa de reinserción social. Santiago, Chile.

Townsend E, Wilcock AA. (2004) Occupational justice and client centred practice: a dialogue in progress. Can J Occup Ther. 71(2):75-87. http://doi.org/5qq. 